\title{
Correction: The Return Trip Is Felt Shorter Only Postdictively: A Psychophysiological Study of the Return Trip Effect
}

\author{
Ryosuke Ozawa, Keisuke Fujii, Motoki Kouzaki
}

\section{Notice of Republication}

This article was republished on June 27, 2015, to correct an error in the title. The title erroneously said "The Return Trip Is Felt Longer" instead of "The Return Trip Is Felt Shorter." Please download this article again to view the correct version. The originally published, uncorrected article and the republished, corrected article are provided here for reference.

\section{Supporting Information}

S1 File. Originally published, uncorrected article.

(PDF)

S2 File. Republished, corrected article.

(PDF)

\section{Reference}

1. Ozawa R, Fujii K, Kouzaki M (2015) The Return Trip Is Felt Shorter Only Postdictively: A Psychophysiological Study of the Return Trip Effect. PLoS ONE 10(6): e0127779. doi: 10.1371/journal.pone. 0127779 PMID: 26061138

\section{OPEnACCess}

Citation: Ozawa R, Fujii K, Kouzaki M (2015) Correction: The Return Trip Is Felt Shorter Only Postdictively: A Psychophysiological Study of the Return Trip Effect. PLoS ONE 10(7): e0133339. doi:10.1371/journal.pone.0133339

Published: July 15, 2015

Copyright: ๑ 2015 Ozawa et al. This is an open access article distributed under the terms of the Creative Commons Attribution License, which permits unrestricted use, distribution, and reproduction in any medium, provided the original author and source are credited. 